\title{
USO DA PODA E DE DIFERENTES DIÂMETROS DE ALPORQUES SOBRE O DESENVOLVIMENTO E O ACÚMULO DE NUTRIENTES DE MUDAS DE LICHIEIRA ${ }^{1}$
}

\author{
CLAUDEMIR FACINCANI FRANCO ${ }^{2}$, RENATO DE MELLO PRADO ${ }^{3}$, LUIZ FERNANDO BRAGHIROLLI², RENATA \\ MOREIRA LEAL ${ }^{2}$, ELIZEU GARCIA PEREZ ${ }^{4}$, LILIANE MAIA ROMUALDO 5
}

\begin{abstract}
RESUMO - O objetivo deste estudo foi avaliar os efeitos do diâmetro do caule e da poda de alporques sobre o desenvolvimento e o acúmulo de nutrientes em mudas de lichieira cultivadas em substrato. Para isso utilizou-se de alporques da variedade Bengal. O delineamento experimental foi o inteiramente casualizado, em esquema fatorial 3 x 2, com oito repetições, sendo três diâmetros do alporque: fino $(1,0 \pm 0,5 \mathrm{~cm}) ;$ médio $(2,0 \pm 0,5 \mathrm{~cm})$ e grosso (3,0 $\pm 0,5 \mathrm{~cm})$, e alporques com e sem poda. As mudas de lichieira foram cultivadas durante 140 dias após o plantio dos alporques. Avaliaramse a matéria seca das brotações da parte aérea e das raízes, e os teores de macro e micronutrientes. A melhor muda de lichieira foi obtida de alporque com diâmetro grosso, e podado. $\mathrm{O}$ alporque de diâmetro médio e fino não-podado, comparado ao podado, proporcionou maior desenvolvimento da muda de lichieira. $\mathrm{O}$ alporque com diâmetro grosso proporcionou maior acúmulo de nutrientes na parte aérea e na raiz da muda de lichieira.
\end{abstract}

Termos para indexação: Litchi chinensis Sonn, alporquia, espessura, macronutrientes, micronutrientes.

\section{USE OF PRUNING AND DIFFERENT AIR LAYING DIAMETERS ON PLANT DEVELOPMENT AND NUTRIENT ACCUMULATION IN LITCHI}

\begin{abstract}
This research was carried out to evaluate the effects of stem diameter and layer pruning on the development and nutrient accumulation in litchi grown on substrate. The variety used here was Bengal. The experiment was arranged in a completely randomized design in a factorial $3 \times 2$ with eight replications, with three layer diameters: thin $(1.0 \pm 0.5 \mathrm{~cm})$; medium $(2.0 \pm 0.5 \mathrm{~cm})$ and thick $(3.0 \pm 0.5 \mathrm{~cm})$ and pruned and not pruned layers. Litchi plants were grown for 140 days after planting the layers. The dry matter of shoot branches and roots and the levels of macro and micronutrients were evaluated. The best litchi plants were obtained from thick, pruned layers. Not pruned layers with medium and thin diameter improved plant development in comparison to pruned ones. Thick layers showed higher nutrient accumulation in shoots and roots.
\end{abstract}

Index terms: Litchi chinensis Sonn, air laying, thickness, macronutrients, micronutrients.

\section{INTRODUÇÃO}

A lichieira (Litchi chinensis Sonn.), família Sapindaceae, tem como centro de origem as regiões subtropicais da China, sendo cultivada há milhares de anos. No Brasil, foi introduzida em 1810 no Rio de Janeiro e, atualmente, seu cultivo vem se expandindo na região Sudeste (Martins, 1998).

Há carência de informações sobre as estatísticas de produção mundial de lichia pela ausência de dados por parte dos principais produtores (Sauco \& Menine, 1987). A produção está restrita a plantas isoladas e a pequenos plantios, atingindo excelentes preços devido à alta procura pelo sabor agradável, além de ser rica em minerais e vitaminas (Martins et al., 2001).

Um dos entraves à sua disseminação é a falta de mudas com qualidade no mercado (Martins, 1998). As mudas de lichieira são produzidas mais por propagação vegetativa, via alporquia, pelo menor período de juvenilidade (5 a 6 anos) e pela obtenção de plantas geneticamente idênticas às matrizes do que por mudas advindas de sementes (Carvalho \& Salomão, 2000). Segundo Abutiate \& Nakasone (1972) e Menzel (1985), o processo de alporquia apresenta dificuldades devido à execução trabalhosa e ao baixo rendimento, uma vez que o processo de obtenção de mudas é lento.

Para a expansão do cultivo da lichieira no Brasil, a obtenção de mudas de alta qualidade é fundamental para garantir a homogeneidade dos pomares. Por isso, os alporques a serem utilizados são importantes, visto afetarem o desenvolvimento da futura muda, uma vez que podem ser de diferentes tamanhos, seja no diâmetro, seja no comprimento. Alguns viveiristas têm utilizado alporques podados, a fim de facilitar o acondicionamento das mudas no viveiro, diminuindo os efeitos da "tortuosidade" dos ramos.

O diâmetro do alporque, e mesmo a presença ou ausência de poda, pode afetar a nutrição das mudas, visto existirem diferenças na biomassa, que poderiam ser fonte de nutrientes para o desenvolvimento das brotações da futura muda. Na literatura, não foram encontradas indicações dos efeitos do manejo dos alporques sobre o desenvolvimento e nutrição das mudas de lichieira.

O objetivo do presente estudo foi de avaliar a influência do diâmetro do ramo e da poda de alporques sobre o desenvolvimento e o acúmulo de nutrientes em mudas de lichieira cultivadas em substrato.

\section{MATERIAL E MÉTODOS}

O experimento foi realizado num viveiro comercial de mudas na fazenda Santo Antônio, no município de Taquaritinga-SP, no período de 31-10-2003 a 17-03-2004. O delineamento experimental foi o inteiramente casualizado, em esquema fatorial $3 \times 2$, com oito repetições, sendo: três diâmetros de alporques: fino $(1,0 \pm 0,5 \mathrm{~cm})$; médio $(2,0 \pm 0,5 \mathrm{~cm})$ e grosso $(3,0 \pm 0,5 \mathrm{~cm})$; com e sem poda. $\mathrm{Na}$ literatura, é indicado usar ramos para alporques com diâmetro de 1,0 a 2,0 cm e de 45 a $65 \mathrm{~cm}$ de comprimento (Carvalho \& Salomão, 2000).

As plantas-matrizes (Litchi chinensis) da variedade Bengal apresentavam cerca de 15 anos quando se realizou a amostragem de folhas ( $2^{\mathrm{a}}$ folha madura) para determinar o estado nutricional. Nesta ocasião, foi realizado o anelamento nos ramos e o envolvimento do ramo em bolsa plástica com turfa para a produção dos alporques. Os alporques foram retirados da planta-matriz, 45 dias após o anelamento, quando as raízes iniciaram a mudança da cor branca para a marromcremosa. Em seguida, foram selecionados os alporques de acordo com o diâmetro e a uniformidade das raízes.

Os alporques foram desfolhados e parte foi podada no ápice e outra não com tesoura de poda, apresentando altura média de 40 e $60 \mathrm{~cm}$, respectivamente. Os alporques foram então colocados em sacos

\footnotetext{
${ }^{1}$ (Trabalho 062/2005). Recebido: 13/03/2005. Aceito para publicação: 17/11/2005.

${ }^{2}$ Mestrando em Ciência do Solo, Depto. de Solos e Adubos, Faculdade de Ciências Agrárias e Veterinárias, Unesp. E-mail: franco@fcav.unesp.br; lfbrag@fcav.unesp.br; rmleal@fcav.unesp.br.

${ }^{3}$ Prof.Dr., Depto. de Solos e Adubos, Faculdade de Ciências Agrárias e Veterinárias, Unesp. Via de Acesso Prof. Paulo Donato Castellane, s/n. 14870-000, Jaboticabal-SP.E-mail: rmprado@fcav.unesp.br; natale@fcav.unesp.br.

${ }^{4}$ Colégio de Post graduandos, Veracruz - México.

${ }^{5}$ Zootecnista, Bolsista CNPq - Apoio técnico.
} 
TABELA 1 - Número médio de folhas e do acúmulo de matéria seca de alporques de mudas de lichieira (Taquaritinga, 2003-2004).

\begin{tabular}{|c|c|c|c|c|}
\hline \multirow{2}{*}{ Fator } & \multirow{2}{*}{ Número de folhas } & \multicolumn{3}{|c|}{ Acúmulo de matéria seca (g) } \\
\hline & & Raízes & Parte aérea & Total \\
\hline \multicolumn{5}{|l|}{ Diâmetro (D) } \\
\hline Grosso & $142,6^{\mathrm{a}}$ & $3,41^{\mathrm{a}}$ & $18,09^{\mathrm{a}}$ & $21,50^{\mathrm{a}}$ \\
\hline Médio & $113,4^{\mathrm{b}}$ & $2,90^{\mathrm{b}}$ & $11,54^{\mathrm{b}}$ & $14,45^{\mathrm{b}}$ \\
\hline Fino & $102,9^{\mathrm{b}}$ & $2,97^{\mathrm{b}}$ & $10,83^{\mathrm{b}}$ & $13,80^{\mathrm{b}}$ \\
\hline \multicolumn{5}{|l|}{ Poda (P) } \\
\hline Com & $97,7^{\mathrm{b}}$ & $2,73^{\mathrm{b}}$ & $11,10^{\mathrm{b}}$ & $13,83^{\mathrm{b}}$ \\
\hline Sem & $141,5^{\mathrm{a}}$ & $3,46^{\mathrm{a}}$ & $15,87^{\mathrm{a}}$ & $19,33^{\mathrm{a}}$ \\
\hline \multicolumn{5}{|l|}{ Teste F } \\
\hline $\mathrm{D}$ & $27,2^{* *}$ & $22,3^{* *}$ & $90,6^{* *}$ & $94,8^{* *}$ \\
\hline $\mathrm{P}$ & $92,5^{* *}$ & $119,3^{* *}$ & $96,5^{* *}$ & $118,1^{* *}$ \\
\hline $\mathrm{D} \times \mathrm{P}$ & $1,1^{\mathrm{ns}}$ & $71,2^{* *}$ & $0,8^{\mathrm{ns}}$ & $0,1^{\mathrm{ns}}$ \\
\hline C. V. $(\%)$ & 13,2 & 7,6 & 12,4 & 10,6 \\
\hline
\end{tabular}

As médias seguidas de letras iguais na vertical não diferem entre si, pelo teste de Tukey, a $5 \%$ de probabilidade.

ns - não significativo; * - Significativo a $5 \%$ de probabilidade; ** - Significativo a $1 \%$ de probabilidade.

plásticos de $5 \mathrm{dm}^{3}$ com substrato Plantmax ${ }^{\circledR}$. A análise química do substrato revelou os valores: $\mathrm{pH}\left(\mathrm{CaCl}_{2}\right)=5,6 ; \mathrm{M} . \mathrm{O} .=161 \mathrm{~g} \mathrm{dm}^{-3}$; $\mathrm{P}_{\text {(resina) }}=370 \mathrm{mg} \mathrm{dm}^{-3} ; \mathrm{K}=5,3 ; \mathrm{Ca}=130 ; \mathrm{Mg}=40 ;(\mathrm{H}+\mathrm{Al})=42 ; \mathrm{SB}=$

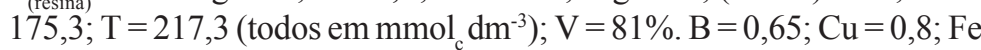
$=260 ; \mathrm{Mn}=8,2 ; \mathrm{Zn}=2,6$ (todos em $\mathrm{mg} \mathrm{dm}^{-3}$ ) (Raij et al., 2001).

As mudas receberam irrigação por microaspersão e foram conduzidas por 140 dias. Avaliaram-se o número de folhas, a matéria seca das brotações da parte aérea e das raízes, e os teores de macro e micronutrientes, utilizando a metodologia descrita por Bataglia et al. (1983). Os resultados foram submetidos à análise de variância, mediante a aplicação de teste F, e a comparação de médias, pelo teste de Tukey $(\mathrm{p}<0,05)$.

\section{RESULTADOS E DISCUSSÃO}

O número de folhas e o acúmulo de matéria seca das brotações da parte aérea, nas raízes e no total das mudas, foram significativamente superiores nos alporques que não receberam poda em relação aos podados. Os alporques com diâmetro de caule grosso apresentaram número de folhas, acúmulo de matéria seca das brotações da parte aérea, nas raízes e no total das mudas, significativamente superiores aos com caule médio e fino (Tabela 1 ).

Para o acúmulo de matéria seca nas raízes, o alporque de diâmetro grosso com poda foi superior ao sem poda, podendo-se inferir que alporques de diâmetro grosso, quando podados, originam mudas com sistema radicular mais vigoroso e desenvolvido. Este resultado está de acordo com Carvalho \& Salomão (2000), que relataram que, quanto mais desenvolvido o ramo em que se faz o alporque, melhor o enraizamento, devido à maior quantidade de reserva. Nos diâmetros médio e fino, os alporques sem poda originaram as mudas com sistema radicular mais desenvolvido (Tabela 2). Este resultado pode auxiliar no manejo de mudas de lichieira nos viveiros, que objetiva a melhoria no desenvolvimento do sistema radicular.

TABELA 2 - Médias de acúmulo de matéria seca (g) nas raízes de alporques de mudas de lichieira (Taquaritinga, 2003-2004).

\begin{tabular}{llll}
\hline & Diâmetro grosso & Diâmetro médio & Diâmetro fino \\
\hline Com poda & $3,60^{\mathrm{Aa}}$ & $2,17^{\mathrm{Bb}}$ & $2,40^{\mathrm{Ab}}$ \\
Sem poda & $3,22^{\mathrm{Bb}}$ & $3,64^{\mathrm{Aa}}$ & $3,54^{\mathrm{Ba}}$ \\
\hline
\end{tabular}

As médias seguidas de letras maiúsculas iguais na vertical e minúsculas na horizontal não diferem entre si, pelo teste de Tukey, a $5 \%$ de probabilidade. ${ }^{\text {ns }}$ - não significativo; * - Significativo a $5 \%$ de probabilidade; ** - Significativo a $1 \%$ de probabilidade.

Especificamente em mudas, o maior volume de raízes é muito importante para garantir o sucesso na instalação do pomar, pelo efeito na maior taxa de pegamento das plantas no campo.Os teores de nutrientes encontrados nas plantas-matrizes (Tabela 3) estão abaixo dos encontrados em pomares com boa produção na África do Sul para K, Ca e B, e acima para N, P e Cu (Carvalho \& Salomão, 2000), e abaixo para $\mathrm{K}$, acima para $\mathrm{N}$ e dentro da faixa para os demais, para a Austrália (Martins et al., 2001). Estas diferenças estão ligadas às condições edafoclimáticas distintas entre os locais.

TABELA 3 - Teores médios de macro e micronutrientes das folhas de lichieira (plantas matrizes) utilizadas para a produção de mudas por alporquia (Taquaritinga, 2003/2004)

\begin{tabular}{|c|c|c|c|c|c|c|c|c|c|c|}
\hline $\mathrm{N}$ & $\mathrm{P}$ & & $\mathrm{Ca}$ & $\mathrm{Mg}$ & $\bar{S}$ & B & $\mathrm{Cu}$ & $\mathrm{Fe}$ & $\mathrm{Mn}$ & $\mathrm{Zn}$ \\
\hline 15,1 & 1.3 & 6,3 & 6,3 & 4,1 & 0.8 & 26 & 11 & 75 & 149 & 16 \\
\hline
\end{tabular}

Os teores de nutrientes na parte aérea das mudas (Tabela 4) estão abaixo dos citados como adequados por Carvalho \& Salomão (2000), para N, K, Ca, Mg e Mn e acima para P, Cu e Fe. De acordo com Martins et al. (2001), os teores encontrados nas mudas estão abaixo daqueles indicados como adequados para $\mathrm{N}, \mathrm{K}, \mathrm{Ca}, \mathrm{Mg}$ e $\mathrm{Mn}$, acima para $\mathrm{Cu}$ e $\mathrm{Fe}$, e dentro da faixa para $\mathrm{P}$ e $\mathrm{Zn}$. Estas diferenças podem afetar a disponibilidade de nutrientes e devem-se às condições edafoclimáticas distintas e à idade da planta, uma vez que os dados da literatura se referem a pomares adultos e em produção.

Os tratamentos empregando alporques com diâmetro grosso foram significativamente superiores aos com diâmetros médio e fino para teores de $\mathrm{P}$, $\mathrm{Ca}$ e $\mathrm{Cu}$, enquanto para o $\mathrm{K}$ esse comportamento foi oposto. Para o N, o tratamento utilizando caule grosso foi melhor que o fino e, para o Fe, o médio foi mais adequado (Tabela 4). Possivelmente, estas diferenças encontradas nos teores de nutrientes se devam à disponibilidade no alporque.

As mudas de alporques podados apresentaram maiores teores de $\mathrm{Ca}$, enquanto para $\mathrm{P}, \mathrm{Fe}$ e $\mathrm{Zn}$ esse comportamento foi oposto. Os teores dos demais nutrientes não apresentaram diferenças em função da poda (Tabela 4). É pertinente salientar que, de forma geral, os teores de nutrientes não foram uma variável adequada para explicar a maior produção de matéria seca, pois as mudas sem poda tiveram maior desenvolvimento (Tabela 1), entretanto sem apresentar maior teor de nutrientes, exceto o $\mathrm{Ca}$ (Tabela 4).

A quantidade de nutrientes acumulada nas mudas de diâmetro grosso foi significativamente superior àquelas de diâmetros médio e fino (Tabela 5). Este fato poderia ser explicado pela maior matéria seca das mudas provenientes de alporque com diâmetro grosso (Tabela 1). Possivelmente, este fato ocorreu porque as plantas têm habilidade de acumular nutrientes, liberando-os depois para os órgãos de crescimento (Menzel et al., 1992). Na literatura, existem relatos de nutrientes que podem ser armazenados na casca de árvores (maçã) como proteínas (Tromp \& Ovaa, 1973). Assim, mudas de lichieira proveniente de alporques com maior acúmulo de nutrientes em seus 
TABELA 4 - Teores médios de macro e micronutrientes na matéria seca das brotações da parte aérea de mudas de lichieira, em função do diâmetro e da poda dos alporques (Taquaritinga, 2003/2004).

\begin{tabular}{|c|c|c|c|c|c|c|c|c|c|c|c|}
\hline Fator & \multicolumn{5}{|c|}{---------------- $\mathrm{g} \mathrm{kg}^{-1}$} & $\bar{S}$ & $\mathrm{~B}$ & $\mathrm{Cu}$ & $\begin{array}{l}\mathrm{Fe} \\
\mathrm{g}^{-1}\end{array}$ & $\mathrm{Mn}$ & $\overline{\mathrm{Zn}}$ \\
\hline \multicolumn{7}{|l|}{ Diâmetro } & \multicolumn{5}{|c|}{ 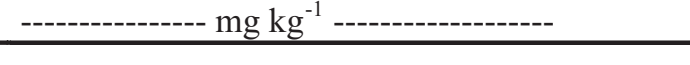 } \\
\hline Grosso & $9,1^{\mathrm{a}}$ & $1,0^{\mathrm{b}}$ & $4,7^{\mathrm{b}}$ & $3,1^{\mathrm{a}}$ & $1,7^{\mathrm{a}}$ & $1,0^{\mathrm{a}}$ & $29^{\mathrm{a}}$ & $19^{\mathrm{b}}$ & $350^{\mathrm{a}}$ & $12^{\mathrm{a}}$ & $25^{\mathrm{a}}$ \\
\hline Fino & $8,2^{b}$ & $1,2^{\mathrm{a}}$ & $5,4^{\mathrm{a}}$ & $2,6^{\mathrm{b}}$ & $1,8^{\mathrm{a}}$ & $1,0^{\mathrm{a}}$ & $31^{\mathrm{a}}$ & $20^{\mathrm{ab}}$ & $344^{\mathrm{a}}$ & $13^{\mathrm{a}}$ & $23^{\mathrm{a}}$ \\
\hline \multicolumn{12}{|l|}{ Poda } \\
\hline Com & $8,6^{\mathrm{a}}$ & $1,2^{\mathrm{a}}$ & $5,2^{\mathrm{a}}$ & $2,8^{\mathrm{a}}$ & $1,8^{\mathrm{a}}$ & $1,1^{\mathrm{a}}$ & $33^{\mathrm{a}}$ & $20^{\mathrm{a}}$ & $359^{\mathrm{a}}$ & $13^{\mathrm{a}}$ & $25^{\mathrm{a}}$ \\
\hline \multicolumn{12}{|l|}{ Teste $\mathbf{F}$} \\
\hline $\mathrm{D}$ & $3,78^{*}$ & $8,45^{* *}$ & $6,41^{* *}$ & $18,30^{* *}$ & $1,34^{\mathrm{ns}}$ & $0,36^{\mathrm{ns}}$ & $1,72^{\mathrm{ns}}$ & $3,03^{\mathrm{ns}}$ & $5,11^{*}$ & $2,18^{\mathrm{ns}}$ & $2,90^{\mathrm{ns}}$ \\
\hline $\mathrm{P}$ & $0,05^{\mathrm{ns}}$ & $4,85^{*}$ & $0,01^{\mathrm{ns}}$ & $6,24^{*}$ & $0,08^{\text {ns }}$ & $5,58^{*}$ & $9,72^{* *}$ & $1,67^{\mathrm{ns}}$ & $13,64^{* *}$ & $1,81^{\mathrm{ns}}$ & $13,76^{* *}$ \\
\hline $\mathrm{DX} P$ & $0,42^{\mathrm{ns}}$ & $0,60^{\mathrm{ns}}$ & $1,98^{\text {ns }}$ & $5,93^{* *}$ & $1,71^{\mathrm{ns}}$ & $0,40^{\text {ns }}$ & $0,33^{\text {ns }}$ & $23,29^{* *}$ & $2,46^{\mathrm{ns}}$ & $9,11^{* *}$ & $2,70^{\mathrm{ns}}$ \\
\hline C. V. $(\%)$ & 10,5 & 16,6 & 15,7 & 18,6 & 17,6 & 15,4 & 15,7 & 11,2 & 17,4 & 13,8 & 15,0 \\
\hline
\end{tabular}

As médias seguidas de letras iguais na vertical não diferem entre si, pelo teste de Tukey, a $5 \%$ de probabilidade.

ns - não significativo; * - Significativo a $5 \%$ de probabilidade; ** - Significativo a $1 \%$ de probabilidade.

TABELA 5 - Quantidade de macro e micronutrientes acumulados na matéria seca das brotações da parte aérea de mudas de lichieira, em função do diâmetro e da poda dos alporques (Taquaritinga, 2003/2004)

\begin{tabular}{|c|c|c|c|c|c|c|c|c|c|c|c|}
\hline Fator & $\mathrm{N}$ & $\mathrm{P}$ & $\mathrm{K}$ & $\mathrm{Ca}$ & $\mathrm{Mg}$ & $\mathrm{S}$ & B & $\mathrm{Cu}$ & $\mathrm{Fe}$ & $\mathrm{Mn}$ & $\mathrm{Zn}$ \\
\hline \multicolumn{11}{|l|}{ Diâmetro } & \\
\hline Grosso & $195,15^{\mathrm{a}}$ & $21,99^{\mathrm{a}}$ & $99,99^{\mathrm{a}}$ & $65,40^{\mathrm{a}}$ & $36,21^{\mathrm{a}}$ & $21,80^{\mathrm{a}}$ & $0,62^{\mathrm{a}}$ & $0,40^{\mathrm{a}}$ & $7,34^{\mathrm{a}}$ & $0,26^{\mathrm{a}}$ & $0,52^{\mathrm{a}}$ \\
\hline Médio & $125,94^{b}$ & $18,44^{\mathrm{b}}$ & $82,29^{\mathrm{b}}$ & $30,12^{\mathrm{b}}$ & $27,31^{\mathrm{b}}$ & $14,98^{\mathrm{b}}$ & $0,46^{\mathrm{b}}$ & $0,31^{\mathrm{b}}$ & $4,16^{\mathrm{b}}$ & $0,18^{\mathrm{b}}$ & $0,31^{\mathrm{b}}$ \\
\hline Fino & $112,68^{b}$ & $16,86^{\mathrm{b}}$ & $74,37^{\mathrm{b}}$ & $36,64^{\mathrm{b}}$ & $25,19^{\mathrm{b}}$ & $13,86^{\mathrm{b}}$ & $0,43^{\mathrm{b}}$ & $0,27^{b}$ & $4,61^{b}$ & $0,17^{\mathrm{b}}$ & $0,31^{\mathrm{b}}$ \\
\hline \multicolumn{12}{|l|}{ Poda } \\
\hline Com & $120,22^{b}$ & $16,80^{\mathrm{b}}$ & $71,90^{\mathrm{b}}$ & $40,64^{\mathrm{b}}$ & $24,74^{\mathrm{b}}$ & $14,94^{\mathrm{b}}$ & $0,45^{\mathrm{b}}$ & $0,28^{\mathrm{b}}$ & $5,03^{b}$ & $0,17^{\mathrm{b}}$ & $0,35^{\mathrm{b}}$ \\
\hline Sem & $168,96^{\mathrm{a}}$ & $21,34^{\mathrm{a}}$ & $99,21^{\mathrm{a}}$ & $47,47^{\mathrm{a}}$ & $34,41^{\mathrm{a}}$ & $18,82^{\mathrm{a}}$ & $0,55^{\mathrm{a}}$ & $0,37^{\mathrm{a}}$ & $5,74^{\mathrm{a}}$ & $0,24^{\mathrm{a}}$ & $0,41^{\mathrm{a}}$ \\
\hline \multicolumn{12}{|l|}{ Teste F } \\
\hline $\mathrm{D}$ & $85,0^{* *}$ & $8,0^{* *}$ & $10,1_{* * *}^{* *}$ & $60,6^{* *}$ & $12,8^{* *}$ & $26,5^{* *}$ & $21,1_{* * *}^{* *}$ & $30,9^{* *}$ & $57,3^{* *}$ & $46,0_{* * *}^{* *}$ & $42,3_{* *}^{* *}$ \\
\hline $\mathrm{P}$ & $77,2^{* *}$ & $18,3^{* *}$ & $32,8^{* *}$ & $6,0^{*}$ & $26,3^{* *}$ & $16,3^{* *}$ & $14,3^{\text {** }}$ & $44,0^{* *}$ & $7,2^{* *}$ & $64,1^{* *}$ & $7,8^{* *}$ \\
\hline $\mathrm{D} X \mathrm{P}$ & $0,4^{\mathrm{ns}}$ & $0,5^{\mathrm{ns}}$ & $3,2^{\mathrm{ns}}$ & $5,6^{* *}$ & $2,2^{\mathrm{ns}}$ & $0,1^{\mathrm{ns}}$ & $0,4^{\mathrm{ns}}$ & $10,7^{* *}$ & $2,8^{\mathrm{ns}}$ & $9,3^{* *}$ & $3,5^{*}$ \\
\hline C. V. $(\%)$ & 13,3 & 19,5 & 19,3 & 21,9 & 22,1 & 19,8 & 17,9 & 15,6 & 17,1 & 13,4 & 19,4 \\
\hline
\end{tabular}

As médias seguidas de letras iguais na vertical não diferem entre si, pelo Teste de Tukey, ao nível de $5 \%$ de probabilidade. ns - não significativo; ${ }^{*}$ - Significativo a $5 \%$ de probabilidade; $* *$ - Significativo a $1 \%$ de probabilidade.

tecidos, poderiam refletir em melhor adaptação no campo após a instalação do pomar.

As mudas sem poda apresentaram acúmulo de nutrientes significativamente superiores às podadas (Tabela 5). Assim, a variável acúmulo de nutrientes foi mais explicada pelo maior acúmulo de matéria seca comparada ao teor de nutrientes. Este fato deve-se ao crescimento das mudas sem a poda ter promovido o efeito diluição dos nutrientes na sua parte aérea. É pertinente acrescentar que, embora a poda facilite o manejo no processo de obtenção de mudas, retira parte da biomassa, diminuindo o fluxo de nutrientes que poderiam ser remobilizados para suprir os órgãos em crescimento da parte aérea e da raiz das mudas.

Pode-se inferir também que as mudas com diâmetro fino a médio, podadas, necessitariam de um tempo maior para compensar o desenvolvimento, comparadas às que não foram podadas. Entretanto, as mudas com diâmetro maior e podadas poderiam ter um período de viveiro mais rápido, indicando que existe uma alternativa para facilitar o manejo no processo de obtenção de mudas.

\section{CONCLUSÕES}

1. A melhor muda de lichieira foi obtida de alporque com diâmetro grosso, e podado.

2. O alporque de diâmetro médio e fino não-podado, comparado ao podado, proporcionou maior desenvolvimento da muda de lichieira.

3. O alporque com diâmetro grosso proporcionou maior acúmulo de nutrientes na parte aérea e na raiz da muda de lichieira.

\section{AGRADECIMENTOS}

À Fazenda Santo Antônio, Taquaritinga-SP, por ter cedido toda a infra-estrutura (viveiro).

\section{REFERÊNCIAS}

ABUTIATE, W. S.; NAKASONE, H. Y. Studies of vegetative propagation of the lychee (Litchi chinensis Sonn.) with special reference to graftage. Ghana Journal Agricultural Science, Ghana, v.51, p. 201-212, 1972.

BATAGLIA, O.C.; FURLANI, A.M.C.; TEIXEIRA, J.P.F.; FURLANI, P.R.; GALLO, J.R. Métodos de análise química de plantas. Campinas: Instituto Agronômico, 1983. 48p. (Boletim Técnico, 78).

CARVALHO, C. M.; SALOMÃO, L.C.C. Cultura da lichieira. Viçosa: UFV, 2000. 38p. (Boletim de extensão, 43).

MARTINS, A.B.G. Enraizamento de estacas enfolhadas de três variedades de lichia (Litchi chinensis Sonn.). 1998. 100f. Tese (Doutorado em Agronomia)- Faculdade de Ciências Agrárias e Veterinárias, Universidade Estadual Paulista, Jaboticabal, 1998.

MARTINS, A.B.G.; BASTOS, D.C.; SCALOPPI JÚNIOR, E.J. Lichieira (Litchi chinensis Sonn.) Jaboticabal: Sociedade Brasileira de Fruticultura, 2001. 48p.

MENZEL, C. M., Propagation of lychee: a review. Scientia Horticulturae, Amsterdan, v.25.n.1.p. 31-48. 1985.

MENZEL, C. M.; SIMPSON, D. R.; HAYDON, G. F. Partitioning of 
nutrients in bearing lychee trees (Litchi chinensis Sonn). Acta Horticulturae, Wageningen, n.321, p. 535-40, 1992

RAIJ, B.van.; ANDRADE, J.C.; CANTARELLA, H.; QUAGGIO, J.A. (Ed.) Análise química para avaliação da fertilidade do solo. Campinas: Instituto Agronômico, 2001. 285p.
SAUCO, V.G.; MENINI, U.G. Lychee cultivation. New York: FAO Plant Production and Protection, 1987. (Paper, 83)

TROMP, J.S.; OVAA, J.C. Spring mobilization of storage nitrogen in apple bark. Physiologia Plantarum, Copenhagen, v.29, p.1-5,1973. 